\title{
Nicotinic Acid Phosphoribosyltransferase Regulates Cancer Cell Metabolism, Susceptibility to NAMPT Inhibitors, and DNA Repair
}

Francesco Piacente ${ }^{1}$, Irene Caffa ${ }^{1}$, Silvia Ravera ${ }^{2}$, Giovanna Sociali ${ }^{3}$, Mario Passalacqua ${ }^{3}$, Valerio G. Vellone ${ }^{4,5}$, Pamela Becherini ${ }^{1}$, Daniele Reverberi ${ }^{5}$, Fiammetta Monacelli ${ }^{1}$, Alberto Ballestrero ${ }^{1,5}$, Patrizio Odetti ${ }^{1,5}$, Antonia Cagnetta ${ }^{1,5}$, Michele Cea ${ }^{1,5}$, Aimable Nahimana ${ }^{6}$, Michel Duchosal ${ }^{6}$, Santina Bruzzone ${ }^{3}$, and Alessio Nencioni, ${ }^{1,5}$

\section{Abstract}

In the last decade, substantial efforts have been made to identify $\mathrm{NAD}^{+}$biosynthesis inhibitors, specifically against nicotinamide phosphoribosyltransferase (NAMPT), as preclinical studies indicate their potential efficacy as cancer drugs. However, the clinical activity of NAMPT inhibitors has proven limited, suggesting that alternative $\mathrm{NAD}^{+}$production routes exploited by tumors confer resistance. Here, we show the gene encoding nicotinic acid phosphoribosyltransferase (NAPRT), a second $\mathrm{NAD}^{+}$-producing enzyme, is amplified and overexpressed in a subset of common types of cancer, including ovarian cancer, where NAPRT expression correlates with a BRCAness gene expression signature. Both NAPRT and NAMPT increased intracellular $\mathrm{NAD}^{+}$levels. NAPRT silencing reduced energy status, protein synthesis, and cell size in ovarian and pancreatic cancer cells. NAPRT silencing sensitized cells to NAMPT inhibitors both in vitro and in vivo; similar results were obtained with the NAPRT inhibitor 2-hydroxynicotinic acid. Reducing NAPRT levels in a BRCA2-deficient cancer cell line exacerbated DNA damage in response to chemotherapeutics. In conclusion, NAPRT-dependent $\mathrm{NAD}^{+}$biosynthesis contributes to cell metabolism and to the DNA repair process in a subset of tumors. This knowledge could be used to increase the efficacy of NAMPT inhibitors and chemotherapy. Cancer Res; 77(14); 3857-69. @2017 AACR.

\section{Introduction}

Alterations in cell metabolism have emerged as one of the hallmarks of cancer that could possibly lead to new targeted therapeutic approaches (1). A strong reliance on a sustained biosynthesis of nicotinamide adenine dinucleotide $\left(\mathrm{NAD}^{+}\right)$is surely to be included among these alterations (2). Features that are commonly encountered in cancer cells and that are thought to underlie their need to support $\mathrm{NAD}^{+}$production include aberrant metabolic processes, increased cell proliferation, and, perhaps

${ }^{1}$ Department of Internal Medicine and Medical Specialties, University of Genoa, Genoa, Italy. ${ }^{2}$ Department of Pharmacy, Biochemistry Lab, Genoa, Italy. ${ }^{3}$ Department of Experimental Medicine, University of Genoa, Genoa, Italy. ${ }^{4}$ Department of Integrated, Surgical and Diagnostic Sciences, University of Genoa, Genoa, Italy. ${ }^{5}$ Ospedale Policlinico San Martino, I.R.C.C.S. per I'Oncologia, Genoa, Italy. ${ }^{6}$ Service and Central Laboratory of Hematology, University Hospital of Lausanne, Lausanne, Switzerland.

Note: Supplementary data for this article are available at Cancer Research Online (http://cancerres.aacrjournals.org/)

F. Piacente and I. Caffa contributed equally to this article.

Corresponding Author: Alessio Nencioni, University of Genoa, V.le Benedetto XV 6, Genoa 16132, Italy. Phone: 39-010-353-8990; Fax: 39-010-353-7989; E-mail: alessio.nencioni@unige.it

doi: 10.1158/0008-5472.CAN-16-3079

(C)2017 American Association for Cancer Research. most importantly, the need to face constant $\mathrm{NAD}^{+}$consumption by $\mathrm{NAD}^{+}$-degrading enzymes involved in DNA repair (e.g., PARP, SIRT6; refs. 3, 4), in cell signaling (e.g., CD38; ref. 5), or in the prevention of apoptosis (e.g., SIRT1, SIRT6; refs. 3, 6, 7). In line with this notion and based on studies from many laboratories, interrupting $\mathrm{NAD}^{+}$biosynthesis has been proposed as a novel strategy in oncology, and the $\mathrm{NAD}^{+}$biosynthetic machinery is considered as a collection of promising targets for the development of anticancer agents (8-10). Nicotinamide phosphoribosyltransferase (NAMPT), the rate-limiting enzyme for $\mathrm{NAD}^{+}$ production from nicotinamide in mammalian cells, was the first $\mathrm{NAD}^{+}$biosynthetic enzyme for which a clear potential as a cancer target was demonstrated with predicted uses in both solid and hematologic malignancies $(9,11-13)$. Unfortunately, the NAMPT inhibitors FK866 and GMX-1777 both failed in clinical trials (14), suggesting that alternative $\mathrm{NAD}^{+}$sources could be available to tumors. For example, one such source is represented by nicotinamide riboside, which is transformed into $\mathrm{NAD}^{+}$ through nicotinamide riboside kinases (NMRK1/2), instead of through NAMPT, and which can be obtained by tumor cells through the degradation of nicotinamide mononucleotide by the ectocellular enzyme, CD73 (15-17).

In the attempt to identify and, possibly, overcome additional mechanisms of resistance to NAMPT inhibitors, we investigated here the role of nicotinic acid phosphoribosyltransferase (NAPRT), which mediates $\mathrm{NAD}^{+}$production from nicotinic acid (NA). 


\section{Materials and Methods}

\section{Cell lines and reagents}

OVCAR-4, OVCAR-5, OVCAR-8, Capan-1, MIA PaCa2, Phoenix, and HEK293T cells were purchased from ATCC (LGC Standards S.r.l.) in March 2015. Cells were passaged for less than 6 months before resuscitation for this study. Mycoplasma testing was routinely done with the MycoAlert Mycoplasma Detection Kit (Lonza Group Ltd.). Last mycoplasma testing was done in September 2016. Cells were characterized by DNA fingerprinting and isozyme detection. All the cell lines were revived every 3 to 4 months. Cells were maintained in RPMI1640 medium supplemented with 10\% FBS and antibiotics. FK866 was obtained from the NIMH Chemical Synthesis and Drug Supply Program. Puromycin, 2-hydroxynicotinic acid, and GMX-1778 were purchased from Sigma Aldrich S.r.l.

\section{Cell doubling time and cell size determination}

Cell doubling time was estimated as in ref. 18. For cell diameter determination, $10^{5}$ cells/well were plated in 6 -well plates and allowed to adhere for 24 hours. When cells were at a confluence of approximately $50 \%$, cells were trypsinized and cell diameter was determined with a handheld cell counter (Bio-Rad TC20) or by flow cytometry (forward scatter, FSC, determination) using a FACSCalibur (Becton Dickinson).

Viability assay and sulforhodamine B culture dish staining

Cell viability and sulforhodamine B (SRB) dish staining were done as described elsewhere (19).

Cell-cycle analysis

Cell-cycle analysis was performed as described elsewhere (19).

\section{Lentiviral transduction}

The lentiviral plasmids encoding for a control scr-shRNA, and for the NAPRT-sh1-5 (plasmid ID: TRCN0000242730, TRCN0000242729, TRCN0000242727, TRCN0000180305, and TRCN0000242731, respectively), were purchased from SigmaAldrich. Lentiviral transductions were done as described in ref. 6.

\section{NAPRT enzymatic activity assay}

The NAPRT enzymatic activity was assayed using different concentrations of NA and PRPP, in the presence or absence of the NAPRT inhibitor 2-HNA as described in ref. 20

\section{Immunoblotting}

Protein lysate generation and immunoblotting were performed as described elsewhere (19). The following antibodies were used: mouse anti-NAPRT (CL0665, ab211529; Abcam), anti-NAMPT (Bethyl Laboratories), anti-poly-ADP ribose (PAR; Calbiochem; Ab- 1 , mouse $\mathrm{mAb}, 10 \mathrm{H}$, cod. AM80), and mouse anti- $\beta$-actin (clone C4; Santa Cruz Biotechnology).

\section{Protein synthesis assays}

Protein synthesis was quantified by the Click-iT HPG Alexa Fluor 488 Protein Synthesis Assay Kit (Thermo Fisher Scientific) and flow cytometry (FACSCalibur; Becton Dickinson) according to the manufacturer's instructions.

\section{Cell fractionation}

Mitochondria and cytosolic fractions from cells were isolated as described elsewhere (21). Ubiquinol-Cytochrome C Reductase
Core Protein 1 (UQCRC1), phosphofructokinase (PFK), histone 3 (H3), NAPRT, and $\beta$-actin protein expression in mitochondrial and cytosolic fractions were detected by immunoblotting using the following antibodies: anti-UQCRCI (Origene; TA800089), anti-PFK, and anti-H3 antibodies (Cell Signaling Technology; 8164 and 9715 , respectively)

\section{$\mathrm{NAD}^{+}$measurement}

The $\mathrm{NAD}^{+}$content of whole cells, isolated mitochondria, or cytosolic fractions was assessed as described in ref. 22.

\section{Confocal and light microscopy}

NAPRT-EGFP cellular location and DNA damage were detected by confocal microscopy as described in ref. 18. The anti- $\gamma \mathrm{H} 2 \mathrm{AX}$ antibody was from Millipore (Ser139, clone JBW301, cod. 05636) and the secondary Alexa Fluor 488-conjugated antibody was from Jackson Immuno Research (cod. 115-546-006). $\gamma \mathrm{H} 2 \mathrm{AX}$ foci were counted using ImageJ-Find Maxima (23). MitoTracker Deep Red was from Thermo Fisher. For light microscopy, cells were imaged at room temperature using with the $\times 20$ magnification of a Leica DMI3000 B microscope. Images were analyzed using Leica Application Suite v3.6.0.

Three-dimensional culture and spheroid analysis

Three-dimensional cell cultures were done as described in ref. 24.

IHC analysis of NAPRT expression in primary ovarian cancer

Twelve random serous high-grade ovarian carcinoma (HGOSC), stage III FIGO were selected from our Institutional database for IHC analysis of NAPRT expression (authorization CER Liguria 058REG2017). Additional methods can be found in Supplementary Methodology.

\section{Gene set enrichment analysis}

We utilized a public gene expression dataset GSE40595 (25) to explore the pathways potentially regulated by NAPRT. We ranked epithelial ovarian cancer samples by their median NAPRT expression levels and then compared their gene expression. GSEA v2.0 (http://www.broad.mit.edu/gsea) was used to identify significantly enriched pathways (26). Gene sets were downloaded from the Broad Institute's MSigDB (http://www.broadinstitute.org/ gsea/index.jsp) and from ref. 27.

\section{Animal experiments}

All animal studies were conducted in accordance with the Institutional Animal Care and Use Committee of the Ospedale Policlinico San Martino, I.R.C.C.S. per l'Oncologia. Six- to 8week-old female BALB/c athymic $\left(\mathrm{nu}^{+} / \mathrm{nu}^{+}\right)$mice were purchased from Charles Rivers Laboratories. Mice were housed in air-filtered laminar flow cabinets with a 12-hour light cycle and food and water ad libitum. Mice were acclimatized for 1 week. In the subcutaneous tumor xenograft models, $5 \times 10^{6}$ OVCAR- 5 or OVCAR-8, or $2 \times 10^{6}$ Capan-1 cells engineered to express the control scr-shRNA or the NAPRT-sh2, or $3.5 \times 10^{6}$ MIA PaCa2 cells transduced with the vector $\mathrm{pBP}$ or with pBP-NAPRT were injected subcutaneously. Treatment was initiated when the tumors appeared as established palpable masses. In each experiment, mice were randomly assigned to be treated for 3 weeks with either vehicle or FK866 (20 mg/kg i.p. twice-a-day for 4 consecutive days every week). Additional methods can be found in Supplementary Materials and Methods. 


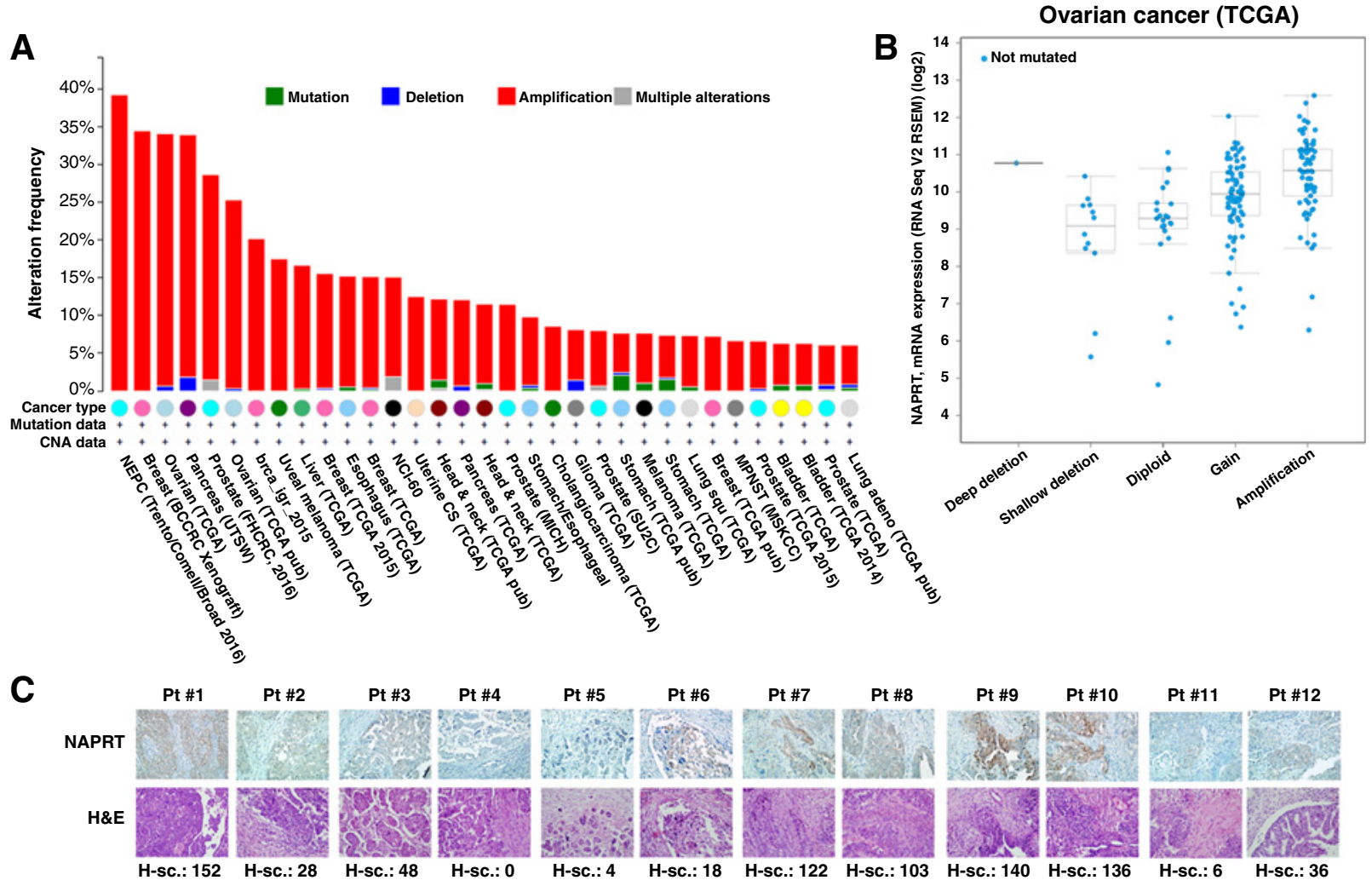

Figure 1.

NAPRT is commonly amplified and overexpressed in solid tumors. A and $\mathbf{B}, N A P R T$ gene mutations, deletions, amplifications, and multiple alterations in human cancer (A), and NAPRT gene expression in ovarian cancer (B) as shown by the cBioPortal for Cancer Genetics (http://www.cbioportal.org/). In A, the cBioPortal for Cancer Genetics search engine was set to highlight studies in which NAPRT was altered in at least 5\% of the tumors. C, NAPRT IHC and hematoxylin-eosin (H\&E) staining in 12 ovarian cancer specimens. H-sc., histology score.

\section{Statistical analyses}

All experiments were repeated at least 3 times. Statistical analyses were performed with GraphPad Prism software version 5 (GraphPad Software). All parameters were tested by paired $t$ test or one-way ANOVA followed by Tukey test. $P$ values $<0.05$ were considered significant.

\section{Results}

NAPRT is overexpressed in a subset of common human solid tumors

We reasoned that the low efficacy of NAMPT inhibitors in patients (14) could reflect the expression by tumors of enzymes that mediate $\mathrm{NAD}^{+}$biosynthesis from precursors other than nicotinamide. If that were the case, a tumor's $\mathrm{NAD}^{+}$pool could be replenished regardless of NAMPT inhibition (17). Aside for nicotinamide, the main $\mathrm{NAD}^{+}$precursors for mammalian cells are represented by the amino acid tryptophan (which is utilized in the so-called de novo $\mathrm{NAD}^{+}$biosynthesis pathway), by NA (utilized in the Preiss-Handler pathway of $\mathrm{NAD}^{+}$biosynthesis), and by nicotinamide riboside (17). The rate-limiting reactions in these $\mathrm{NAD}^{+}$biosynthetic routes are catalyzed by quinolinate phosphoribosyltransferase (QPRT), NAPRT, and NMRK1/2, respectively (17). To gain insight into the expression of these enzymes in human cancer, we used the cBio Cancer Genomics Portal, an open platform that allows exploring multidimensional cancer genomics data $(28,29)$. QPRT, NAPRT, and NMRK1/2 all showed a tendency to be amplified in at least one cancer study (Fig. 1A; Supplementary Fig. S1A). However, among the four enzymes, NAPRT exhibited the most striking frequency of amplification: $>25 \%$ of the cases of ovarian, pancreatic, and prostate cancer, and $>10 \%$ of breast, liver, esophagus, and head and neck cancer (Fig. 1A). NAPRT was normally amplified in a nonmutated form, and its amplification was typically associated to a marked increase in gene expression (Fig. 1B; Supplementary Fig. S1B-S1E). Figure 1C shows NAPRT expression and hematoxylin-eosin staining in a series of 12 primary epithelial ovarian cancers that were referred to our Institution. Consistent with the data from the cBio Cancer Genomics Portal, in this series, four cases were found to express high NAPRT levels (mean histology score, H-score, >120), seven cases exhibited low NAPRT expression (mean H-score <50), whereas one case had medium NAPRT expression (mean H-score $=100$ ). Additional IHC studies of NAPRT in ovarian cancer, as well as in other types of cancer, can be found in the Human Protein Atlas database (http://www.proteinatlas.org/ ENSG00000147813-NAPRT/tissue; refs. 30, 31). Interestingly, by using the NCI-60 Proteome Resource (32), we verified that among all of the cell lines from NCI-60 cell line panel, the ovarian cancer cell lines were those expressing the highest NAPRT levels (proteomics.wzw.tum.de/nci60/). Specifically, OVCAR-5, -4, and -8 

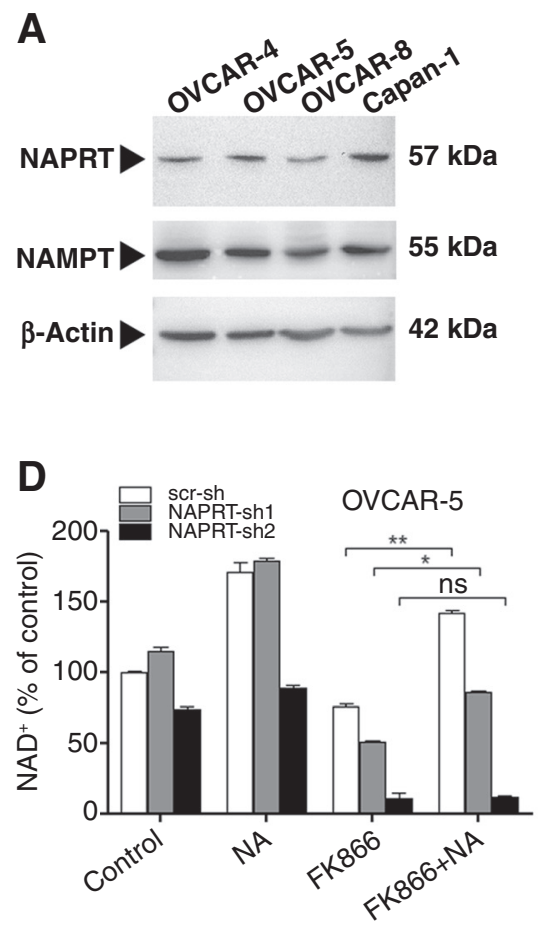

B
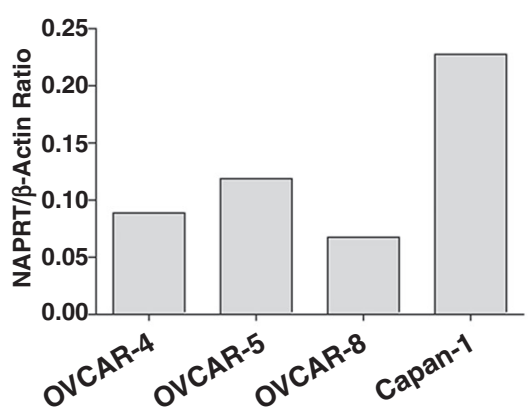

E

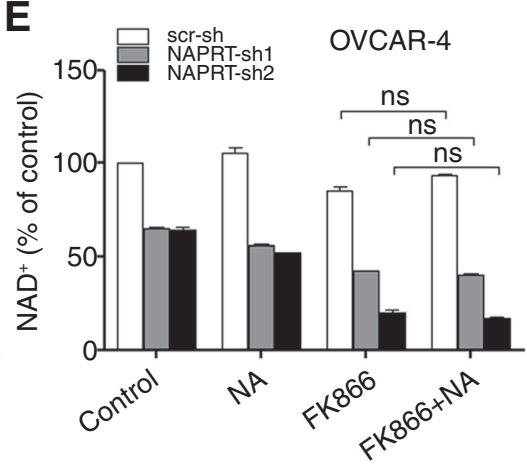

H

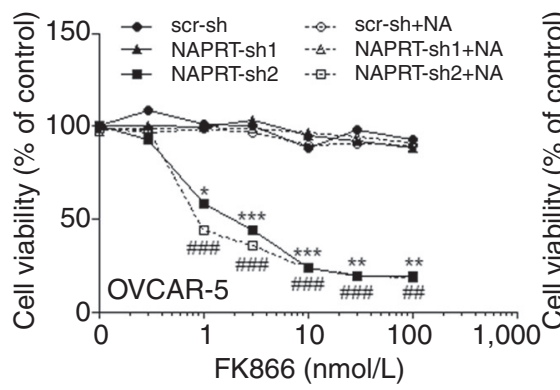

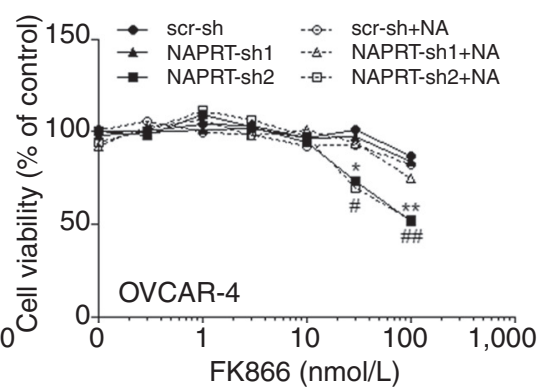

C

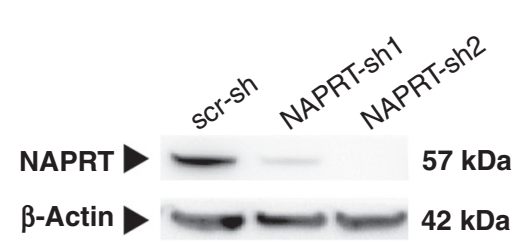

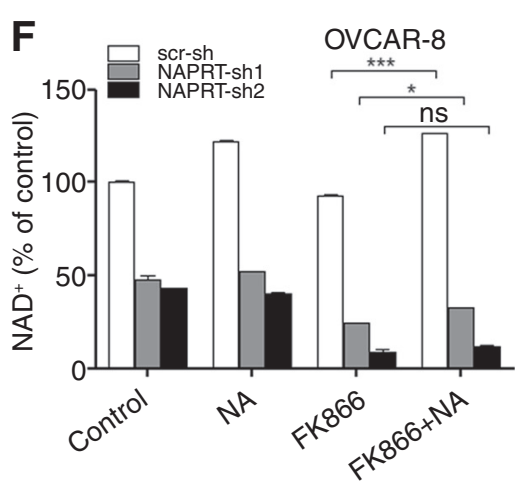

I

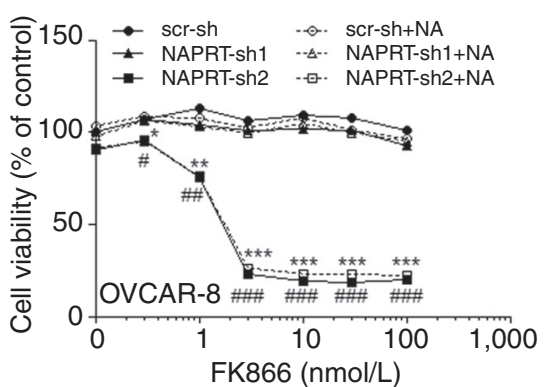

Figure 2.

NAPRT silencing reduces intracellular NAD ${ }^{+}$content and sensitizes ovarian cancer cells to FK866. A and B, OVCAR-5, OVCAR-4, OVCAR-8, and Capan-1 cells were used for protein lysate generation, and NAPRT, NAMPT, and $\beta$-actin levels were determined by immunoblotting. In B, NAPRT band intensity was normalized to $\beta$-actin expression. C, OVCAR-5 were transduced with either one of two anti-NAPRT shRNAs or with a scrambled shRNA. Cells were subsequently used for cell lysate generation, and NAPRT and $\beta$-actin were detected by immunoblotting. D-F, $1 \times 10^{5}$ OVCAR-5, OVCAR-4, and OVCAR-8 cells expressing the scr-shRNA, NAPRT-sh1, or NAPRT-sh2/well were plated in 6-well plates, allowed to adhere overnight, and then stimulated for 24 hours with or without 100 nmol/L FK866 in the presence or absence of $10 \mu \mathrm{mol} / \mathrm{L}$ NA. Thereafter, cells were lysed in $0.6 \mathrm{~mol} / \mathrm{L} \mathrm{PCA}$ and used for NAD ${ }^{+}$determination. G-I, $2 \times 10^{3}$ scr-shRNA-, NAPRT-sh1-, or NAPRT-sh2-expressing OVCAR-5, OVCAR-4, and OVCAR-8 cells/well were plated in 96-well plates, allowed to adhere overnight, and then incubated for 72 hours with or without FK866 at the indicated concentrations in the presence or absence of $10 \mu \mathrm{mol} / \mathrm{L}$ NA. Subsequently, cell viability was detected with SRB. A-C, One representative experiment out of three is presented. D-F, Data are presented as mean \pm SD of three separate experiments. G-I, Data are presented as mean of three separate experiments. ns, nonsignificant; ${ }^{*}, P<0.05 ;{ }^{* *}, P<0.01{ }^{* * *}, P<0.001 ; \#, P<0.05 ; \# \#, P<0.01$; and \#\#\#, P<0.001. In G-I, hashtag symbols refer to the statistical significance of the NAPRT-sh2+NA samples.

emerged as the ovarian cancer cell lines expressing the highest NAPRT amounts.

NAPRT-overexpressing cancer cells are insensitive to FK866

We confirmed that the ovarian cancer cell lines OVCAR-5, -8, and -4 all express both NAPRT and NAMPT (Fig. 2A and B), the highest NAPRT amounts being typically detected in OVCAR-5. In addition, a high NAPRT expression was also found in the pancreatic ductal adenocarcinoma cell line, Capan-1 (Fig. 2A and B). We initially transduced these cell lines with two independent,
NAPRT-targeting shRNAs. Both of the shRNAs reduced NAPRT at the mRNA and protein level, although NAPRT-sh2 was generally more effective (Fig. 2C; Supplementary Fig. S2A-S2D). Notably, no compensatory increase in NAMPT expression in response to NAPRT silencing was detected (Supplementary Fig. S2E-S2H). Thereafter, we monitored total $\mathrm{NAD}^{+}$content and cell viability in baseline conditions, in the presence of the NAMPT inhibitor, FK866, and of NA. NAPRT silencing with NAPRT-sh2 avoided the NA-induced increase in intracellular $\mathrm{NAD}^{+}$levels, that we detected in every cell line, but in OVCAR-4, and strongly increased 
FK866-induced $\mathrm{NAD}^{+}$depletion (Fig. 2D-F; Supplementary Fig. $\mathrm{S} 3 \mathrm{~A})$. Baseline total $\mathrm{NAD}^{+}$levels were also reduced by NAPRTsh2, although to an extent that varied between cell lines based on their baseline NAPRT protein levels: in OVCAR-5 and Capan-1 cells, which started with the highest NAPRT amounts (and which were likely to be left with some residual NAPRT after silencing), $\mathrm{NAD}^{+}$levels were only decreased by NAPRT-sh 2 by $5 \%$ to $25 \%$ (Fig. 2D; Supplementary Fig. S3A). In OVCAR-4 and OVCAR-8 cells, which had lower baseline NAPRT expression and were likely to be virtually devoid of this enzyme after silencing, a more substantial decrease in $\mathrm{NAD}^{+}$could be observed (Fig. $2 \mathrm{E}$ and F). Similarly, NAPRT-sh1, which was less effective in terms of NAPRT silencing compared with NAPRT-sh2 (Fig. 2C; Supplementary Fig. S2A-S2D), reduced baseline $\mathrm{NAD}^{+}$levels in OVCAR4 and OVCAR-8, but not in OVCAR-5 and in Capan- 1 cells (Fig. 2D-F; Supplementary Fig. S3A). This shRNA was also generally less effective at enhancing FK866-induced NAD ${ }^{+}$depletion compared with NAPRT-sh2 (Fig. 2D-F; Supplementary Fig. S3A).

When not combined with one another, FK866 and NAPRT silencing both failed to affect OVCAR-4, $-5,-8$, and Capan- 1 cell viability (Fig. 2G-I; Supplementary Fig. S3B-S3D), which is in line with the intracellular $\mathrm{NAD}^{+}$measurements and with previous studies showing that $\mathrm{NAD}^{+}$reductions that do not reach $90 \%$ to $95 \%$ of the basal content can be tolerated by mammalian cells in terms of their survival (22). However, combining NAPRT silencing by NAPRT-sh2 with FK866 resulted in a marked reduction in cell viability in all of the cell lines tested (Fig. 2G-I; Supplementary Fig. S3B-S3D). In addition, again consistent with the intracellular $\mathrm{NAD}^{+}$measurements, NAPRT silencing with NAPRT-sh2 also abolished the ability of NA supplementation to rescue cancer cells from FK866's cytotoxicity (Fig. 2G-I; Supplementary Fig. S3B). To rule out the possibility that the ability of NAPRT-sh 2 to sensitize NAPRToverexpressing cancer cells to the cytotoxic effect of FK866 could reflect off-target effects of this shRNA, we tested additional shRNAs against NAPRT, NAPRT-sh3, -4 , and -5 (Supplementary Fig. S3E and S3F). Using the OVCAR-5 cell model, we found that NAPRT-sh3 and NAPRT-sh4, which, after NAPRTsh2, were the most effective at silencing NAPRT, also increased the cytotoxic activity of FK866 (Supplementary Fig. S3G), albeit to a lesser extent compared with NAPRT-sh2 (which was by far the most active shRNA, virtually abolishing NAPRT expression). Notably, OVCAR-5 cell sensitization to GMX-1778, another NAMPT inhibitor (33), by NAPRT-sh2, -3 , and -4 was also observed (Supplementary Fig. S3H).

Altogether, these data showed that NAPRT silencing effectively restores the anticancer activity of NAMPT inhibitors in NAPRToverexpressing cancer cells. However, likely due to the fact that NAPRT is highly effective at increasing NAD ${ }^{+}$levels $(20,34)$, its near-complete knockdown is required to achieve sensitization to FK866 and related drugs.

NAPRT reduces DNA damage and regulates cytosolic and mitochondrial $\mathrm{NAD}^{+}$levels, oxidative phosphorylation, and protein synthesis in cancer cells

The tumor types in which we found NAPRT to be most commonly amplified (ovarian, pancreatic, prostate, but also breast cancer) are also those that most frequently exhibit defects in their homologous recombination DNA repair (HRR), a condition also known as BRCAness (35). These tumors are critically reliant on $\mathrm{NAD}^{+}$-consuming enzymes, such as PARP1/2, for their
DNA repair. Thus, we hypothesized that tumors with defective HRR may use NAPRT-mediated $\mathrm{NAD}^{+}$production to fuel their DNA repair mechanisms, including PARP activity. To test this hypothesis, we assessed gene expression profiles in a set of epithelial ovarian cancers based on their NAPRT levels, using GSEA (26). By this approach, we found the gene expression profile of BRCAness identified by Konstantinopoulos and colleagues (27) to significantly correlate with NAPRT expression (FDR = $0.23 ; P<0.05$; Fig. 3A), whereas the KEGG DNA Damage Response Signal Transduction gene set was found to exhibit borderline correlation with high-NAPRT expression (FDR = $0.25 ; P=0.05 ;$ Fig. 3B). We subsequently assessed the role of NAPRT in the susceptibility to DNA damage of Capan-1, which are considered a model of BRCA2-deficient cancer cells (36). In the first place, using Western blotting (37), we detected reduced levels of PAR in NAPRT-silenced Capan-1 (Fig. 3C), which is consistent with a reduced PARP activity. Thereafter, we found that the alkylating agent, MMS, and the chemotherapeutic, doxorubicin, caused more DNA damage in NAPRT-silenced Capan-1 than in control cells, as detected based on counts of $\gamma \mathrm{H} 2 \mathrm{AX}$ foci (Fig. 3D and E). Supplementation with exogenous $\mathrm{NAD}^{+}$reduced MMS-mediated DNA damage in NAPRT-silenced Capan-1 (Fig. 3D and E), although with a lower efficiency compared with the control cells. Finally, consistent with their increased predisposition to DNA damage, NAPRT-silenced Capan-1 cells showed an impaired capacity to form colonies after a transient exposure to MMS (Fig. 3F and G). Notably, here, even NAPRT silencing with the less efficient shRNA, NAPRT-sh1, was sufficient to increase Capan-1 susceptibility to MMS, suggesting that the DNA repair process of this BRCA2-deficient cell line is critically reliant on high NAPRT levels. Altogether, these data are in line with NAPRT playing a role in the DNA repair mechanisms of cancer cells with defective HRR.

We subsequently focused on the role of NAPRT in cancer cell metabolism. In the first place, we performed subcellular fractionation, isolating mitochondria and cytosol from OVCAR-4, OVCAR-5, OVCAR-8, and Capan-1 cells expressing a control shRNA or NAPRT-sh2, and measured the NAD ${ }^{+}$content of these fractions. In OVCAR- $4,-5$, and -8 cells, NAPRT silencing reduced both the cytosolic and the mitochondrial $\mathrm{NAD}^{+}$pools (Fig. 4A and $\mathrm{B}$ ). Conversely, in Capan-1 cells, only the mitochondrial $\mathrm{NAD}^{+}$levels were blunted by NAPRT silencing, suggesting a specific reliance of these organelles on NAPRT for the maintenance of their $\mathrm{NAD}^{+}$stores. A previous study indicated that NAPRT is primarily located in the cytosol and in the nucleus, but not in the mitochondria (38). Consistent with this report, we could readily detect endogenous NAPRT protein in cytosolic, but not in mitochondrial cell fractions from OVCAR-4, -5, -8, and Capan-1 cells (Supplementary Fig. S4A). We generated an EGFPtagged human NAPRT and transduced it into OVCAR-5 and Capan-1. In these cell lines, NAPRT-EGFP exhibited a diffuse cytoplasmic and nuclear location (Fig. 4C and D). However, no clear mitochondrial location could be demonstrated. Thus, our findings are in line with those of Nifikorov and colleagues (38) and indicate that NAPRT is probably not present in mitochondria and that NAPRT-derived nucleotides are likely to fuel the mitochondrial $\mathrm{NAD}^{+}$pool after import from the cytoplasm.

In subsequent experiments, we determined the effect of NAPRT silencing with or without concomitant NAMPT inhibition with FK866 on oxidative phosphorylation (OXPHOS), glycolysis, and energy status of OVCAR-5 cells. Consistent with the marked 
A

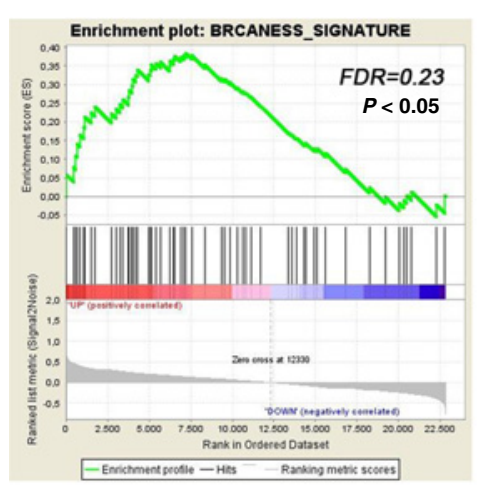

B

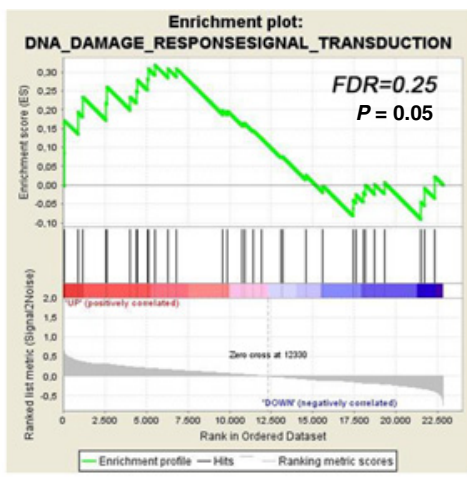

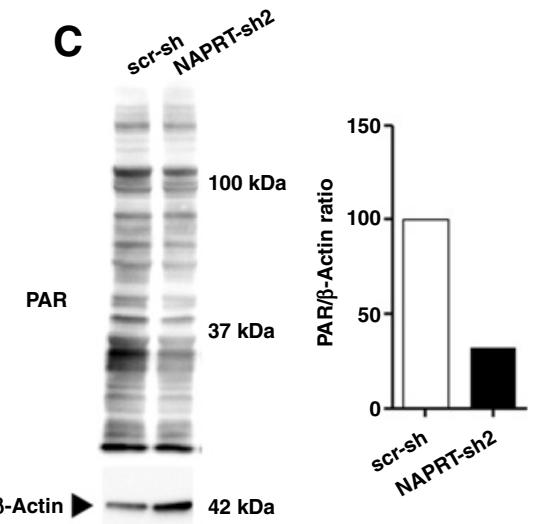

E

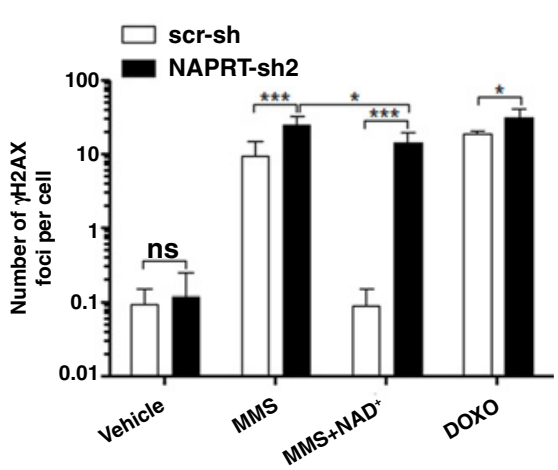

$\beta$-Actin

$42 \mathrm{kDa}$

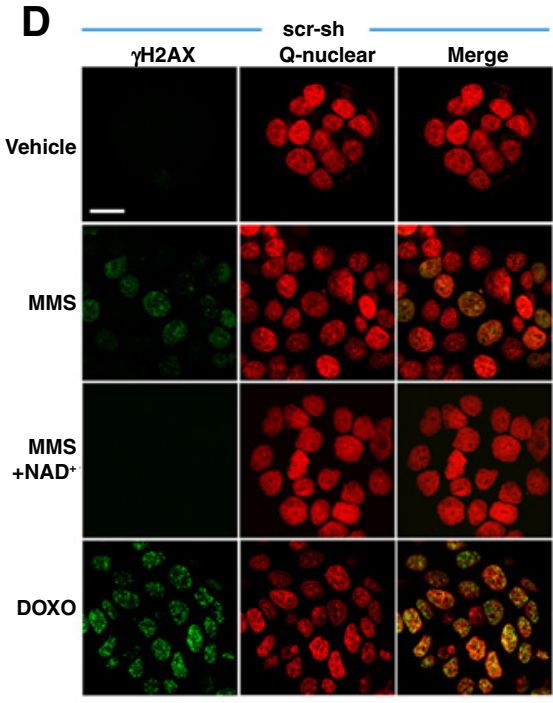

F

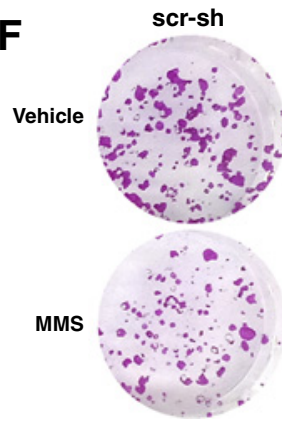

NAPRT-sh1

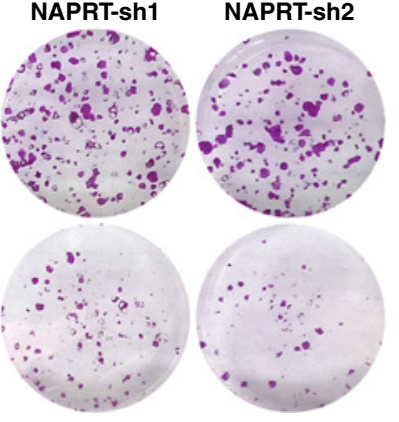

NAPRT-sh2
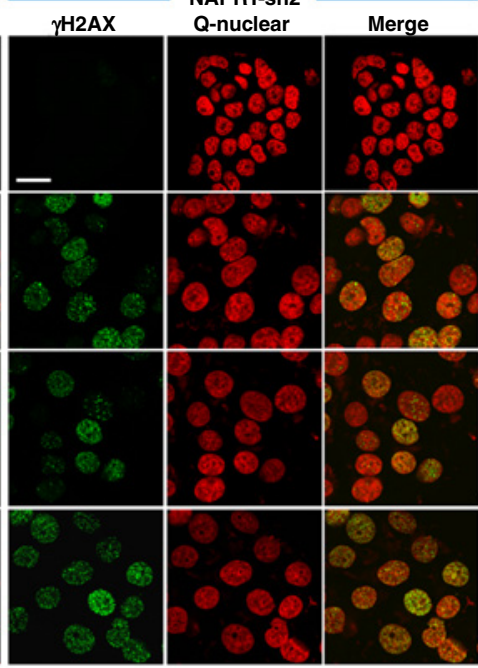

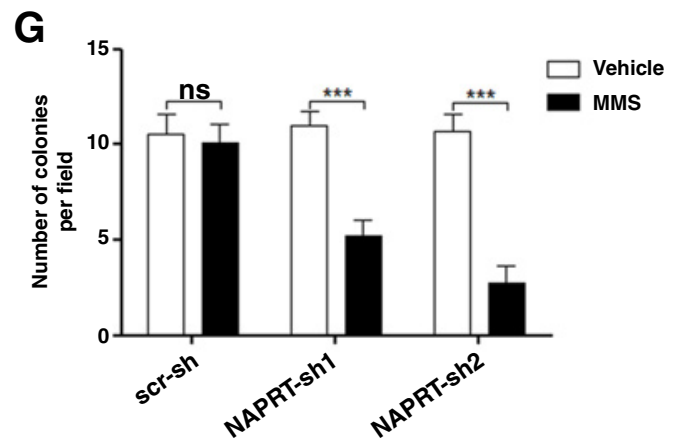

Figure 3.

NAPRT promotes DNA repair in cancer cells with defective HRR. A and B, The pathways potentially regulated by NAPRT were explored by GSEA in the public gene expression data set, GSE40595. Epithelial ovarian cancer samples were ranked by their median NAPRT expression levels, and GSEA was used to identify significantly enriched pathways. C, Capan-1 cells were transduced with NAPRT-sh2 or with scr-shRNA. Cells were subsequently used for cell lysate generation, and PAR and $\beta$-actin were detected by immunoblotting. D and E, $2 \times 10^{4}$ Capan-1 cells transduced with a scr-shRNA or with the NAPRT-sh2/well were plated in tissue culture chambers. Cells were allowed to adhere overnight and were subsequently treated for 1 hour with or without 1 mmol/L MMS or $3 \mu \mathrm{mol} / \mathrm{L}$ doxorubicin, in the presence or absence of $100 \mu \mathrm{mol} / \mathrm{L} \mathrm{NAD}^{+}$. Thereafter, cells were washed with PBS, fixed, permeabilized, and $\gamma \mathrm{H} 2 \mathrm{AX}$ foci and nucle (Q-nuclear) were visualized by confocal microscopy (D); scale bar, $20 \mu \mathrm{m}$. The number of $\gamma \mathrm{H} 2 \mathrm{AX}$ foci per cell/nucleus was quantified by counting at least 50 nuclei per treatment condition (E). F and G, $1 \times 10^{3}$ Capan-1 cells transduced with a scr-shRNA or with the NAPRT-sh1 or -sh2/well were plated in 6 -well plates. Cells were allowed to adhere overnight and were subsequently treated with $1 \mathrm{mmol} / \mathrm{L}$ MMS for 1 hour. Thereafter, cells were washed and incubated in regular culture medium for 2 weeks. Finally, the plates were stained with SRB and the colonies/well were counted using standard microscopy. C, D, and F, One representative experiment out of three is presented. E and $\mathbf{G}$, Results are mean $\pm \mathrm{SD}$ of three separate experiments. ns, nonsignificant; ${ }^{*}, P<0.05 ;$ and ${ }^{* * *}, P<0.001$. 
A

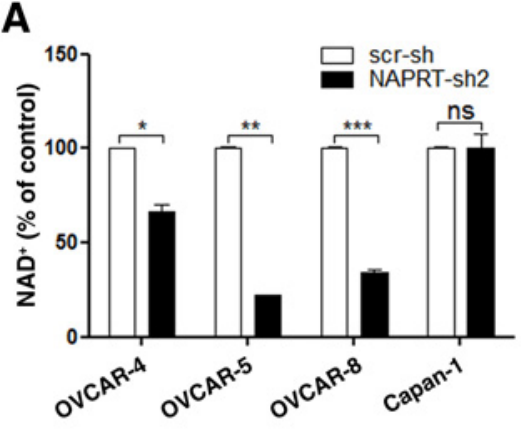

D

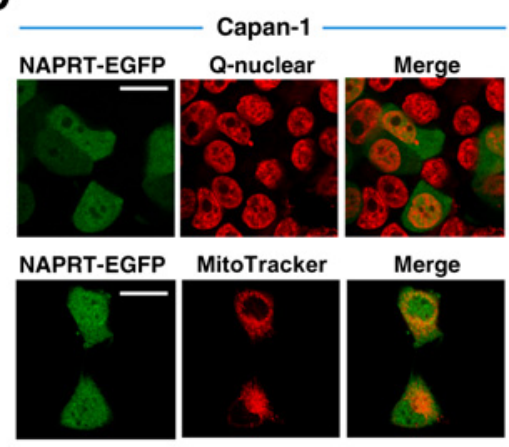

G

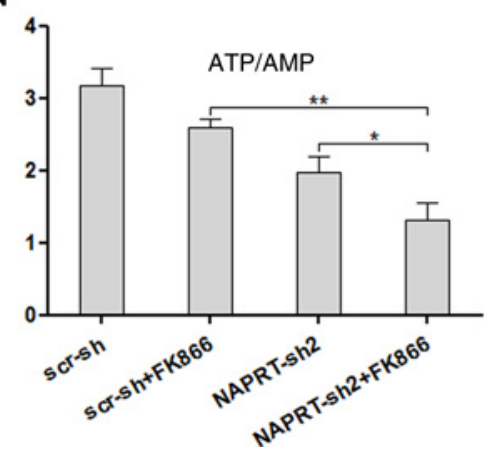

B

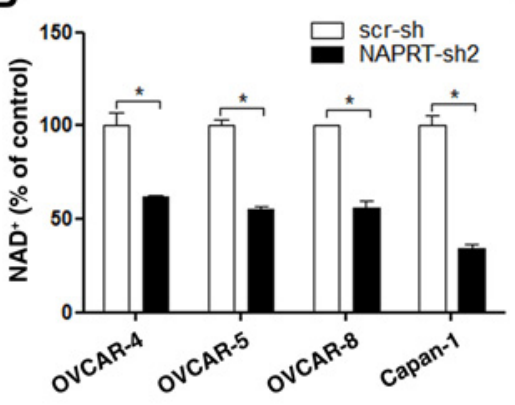

$E$

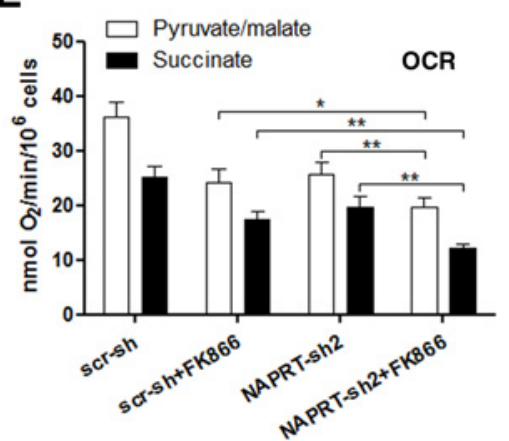

H

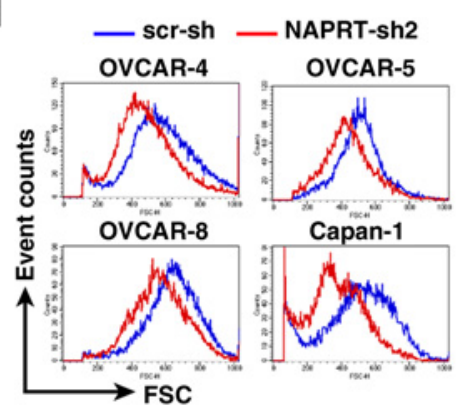

C

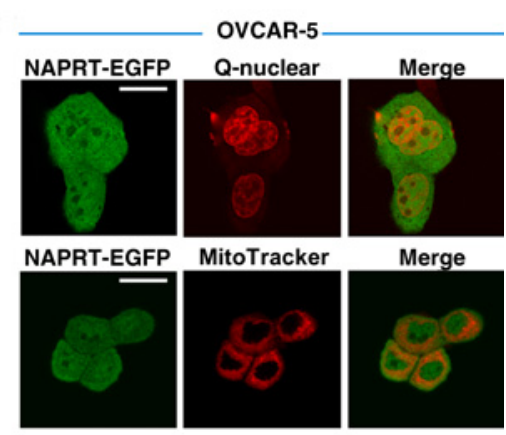

F

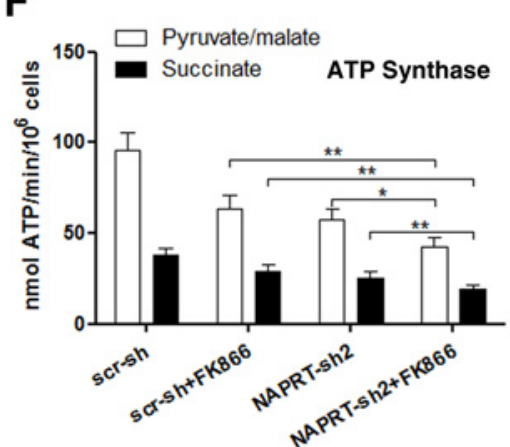

I

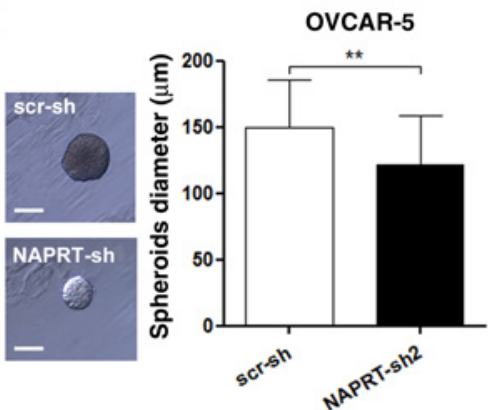

Figure 4.

NAPRT regulates cytosolic and mitochondrial NAD ${ }^{+}$levels, OXPHOS, and energy status in cancer cells. A and B, $2 \times 10^{6}$ scr-shRNA- or NAPRT-sh2-expressing OVCAR-4, OVCAR-5, OVCAR-8, and Capan-1 cells/flask were seeded in $175 \mathrm{~cm}^{2}$ flasks, allowed to grow up to $80 \%$ confluence, and subsequently used for the isolation of mitochondria and cytosolic fractions. NAD ${ }^{+}$levels in both fractions were subsequently determined and normalized to protein content of each fraction. $\mathbf{C}$ and $\mathbf{D}$, OVCAR-5 and Capan-1 cells were retrovirally engineered to express a NAPRT-EGFP fusion protein. Then, NAPRT-EGFP location was determined by confocal microscopy after cell staining with a fluorescent dye that stains mitochondria (MitoTracker Deep Red) or a dye that stains nuclei (Q-Nuclear); scale bars, $15 \mu \mathrm{m}$. E-G, $3 \times 10^{6}$ OVCAR- 5 cells expressing the scr-shRNA or NAPRT-sh2/flask were seeded in $75 \mathrm{~cm}^{2}$ flasks, allowed to adhere overnight, and then stimulated for 24 hours with or without $100 \mathrm{nmol} / \mathrm{L}$ FK866. Thereafter, cells were used for the generation of lysates in which OCR, ATP synthase activity, as well as ATP/AMP ratio were determined. H, OVCAR-4, OVCAR-5, OVCAR-8, and Capan-1 cells were analyzed by FACS for estimating their FSC as a correlate of their volume. Note that $1 \times 10^{4}$ events per cell line were recorded. I, OVCAR- 5 cells expressing either the scr-shRNA or the NAPRT-sh2 were used for three-dimensional cultures. Ten days after cell seeding, the spheroids arising from the single cells were imaged by light microscopy and their diameter was measured; scale bars, $100 \mu \mathrm{m}$. A, B, E, F, G, and I (histogram), Data are presented as mean \pm SD of three separate experiments. ns, nonsignificant; ${ }^{*}, P<0.05$; ** $P<0.01 ;{ }^{* * *}, P<0.001$. In E-G, the experimental values for scr-sh+FK866, NAPRT-sh2, and NAPRT-sh2+FK866 all had $P<0.05$ compared with those for scr-sh. C, D, H, and I (photographs), One representative experiment out of three is presented.

reduction in the total and, especially, in the mitochondrial $\mathrm{NAD}^{+}$ pool observed in NAPRT-silenced OVCAR-5, a significant decrease in oxygen consumption rate (OCR), indicative of a decreased oxidative metabolism, could be observed in these cells compared with the control cells (Fig. 4E). This corresponded to a significant reduction in ATP synthase activity (Fig. 4F). FK866 also blunted OCR and ATP synthase activity, whereas combined
NAPRT silencing and FK866 treatment achieved the most pronounced OCR and ATP synthesis reduction (Fig. 4E and F) Notably, the P/O ratio was not affected by NAPRT silencing, FK866, or by their combination, indicating that none of these treatments has uncoupling effects (Supplementary Fig. S4B). The ATP/AMP ratio was reduced by NAPRT silencing, by FK866 and, to the highest extent, by the two combined interventions (Fig. 4G; 
Table 1. NAPRT silencing reduces protein synthesis and cell size in cancer cells

\begin{tabular}{lccc}
\hline Cell line & $\begin{array}{c}\text { Doubling } \\
\text { time }(\mathbf{h})\end{array}$ & $\begin{array}{c}\text { Cell diameter } \\
(\boldsymbol{\mu} \mathbf{g} / \mathbf{m L})\end{array}$ & $\begin{array}{c}\text { Normalized mean } \\
\text { Click-iT HPG signal }\end{array}$ \\
\hline OVCAR-5 scr-sh & 28 & 15.2 & 1 \\
OVCAR-5 NAPRT-sh2 & 30 & 14.1 & 0.7 \\
OVCAR-8 scr-sh & 27 & n.a. & n.a. \\
OVCAR-8 NAPRT- & 31 & n.a. & n.a. \\
$\quad$ sh2 & & & 1 \\
Capan-1 scr-sh & 31 & 15.2 & 0.54 \\
Capan-1 NAPRT-sh2 & 36 & 14.7 & \\
\hline
\end{tabular}

NOTE: OVCAR-5, OVCAR-8, and Capan-1 cells were utilized for estimating thei doubling times, cell diameter, and protein synthesis. Within each cell line, the Click-iT HPG signal obtained (mean fluorescence intensity as detected by flow cytometry) was normalized to the signal produced by the same, cycloheximidetreated cells. Thereafter, the values obtained in NAPRT-silenced cells were normalized to those obtained in control cells (cells expressing the scrambled shRNA).

Abbreviation: n.a., not assessed.

Supplementary Fig. S4C and S4D). Notably, the activity of glycolytic enzymes in cells with silenced NAPRT was not reduced. In fact, in NAPRT-silenced OVCAR-5, increased hexokinase, phosphofructokinase, pyruvate kinase, and lactate dehydrogenase activities were typically detected (Supplementary Fig. S4E-S4H).

NAPRT silencing had minor effects on the ability of cancer cells to proliferate, because cell doubling time was only increased by $5 \%$ to $20 \%$ in OVCAR-5, OVCAR- 8 , and Capan1 cells expressing the NAPRT-sh2 (Table 1). Cell-cycle studies of OVCAR-5 and Capan-1 cells showed a slight reduction in the representation of cells in the $G_{1}$ phase among the NAPRTsilenced cells (Supplementary Fig. S4I). FK866 had no effect on the cell-cycle profile of control cells, but, consistent with the viability data, it increased the representation of hypodiploid nuclei (indicative of ensuing cell death) in cells expressing NAPRT-sh2 (Supplementary Fig. S4I).

Interestingly, likely as a result of their impaired energy status, NAPRT-silenced cells were invariably smaller compared with the control cells as detected by measurements of their diameter and by flow cytometry (Table 1 and Fig. 4H), and this correlated with a reduced ability to perform protein synthesis (Table 1 ).

In three-dimensional Matrigel cultures, both OVCAR- 5 cells expressing the control shRNA and OVCAR-5 expressing NAPRTsh2 gave rise to spheroids that grew gradually with a typical spherical shape (Fig. 4I). No effect of NAPRT silencing on the number of spheroids obtained with the two cell types could be detected (data not shown). However, in line with their smaller cell size and with their slightly increased doubling time, NAPRT-sh2expressing OVCAR-5 gave rise to spheroids that were typically smaller than the controls (Fig. 4I).

Therefore, in NAPRT-overexpressing cancer cells, NAPRT plays a pivotal role in the regulation of cellular $\mathrm{NAD}^{+}$stores, OXPHOS, energy status, protein synthesis, and cell size.

\section{NAPRT inhibition with 2-hydroxinicotinic acid reduces OXPHOS in NAPRT-overexpressing carcinoma cells and sensitizes them to FK866}

Previous studies indicated the ability of structural analogues of NA to inhibit NAPRT enzymatic activity (39-41). Using the enzymatic approach established by Galassi and colleagues (20), we confirmed a significant inhibition of NAPRT activity by 2-hydroxinicotinic acid (2-HNA), as detected by HPLC quantification of the NAPRT product, NA mononucleotide (Supplementary Fig. S5A-S5C). Consistent with these data, combining 2-HNA with FK866 in OVCAR-5 cells resulted in a more pronounced $\mathrm{NAD}^{+}$depletion compared with FK866 alone (Fig. 5A). 2-HNA effectively sensitized OVCAR- 5 and Capan-1 cells to FK866 in terms of cell viability (Fig. 5B and C), recreating the effect of NAPRT silencing with NAPRT-sh2 (Fig. 2G; Supplementary Fig. S3B), and it also sensitized OVCAR-5 cells to GMX-1778 (Supplementary Fig. S5D). Similar to NAPRT silencing, 2-HNA also reduced OCR and ATP synthase activity, which translated into a reduction in the ATP/AMP ratio, and these effects were further compounded by FK866 addition (Fig. 5D, E, G-I). 2-HNA, FK866, and their combination failed to affect the P/O ratio (Fig. $5 F$ ). Finally, the effect of 2-HNA on the activity of glycolytic enzymes mirrored that obtained by NAPRT silencing, as this compound also increased hexokinase, phosphofructokinase, pyruvate kinase, and lactate dehydrogenase activities (Supplementary Fig. S5E-S5H).

To formally show that NAPRT overexpression confers resistance to FK866 to a cancer cell line that is normally sensitive to this NAMPT inhibitor and to test the effect of 2-HNA in such a model, we engineered the pancreatic carcinoma cell line MIA PaCa2 to overexpress NAPRT (Supplementary Fig. S6A). In baseline conditions, MIA PaCa2 cells expressed NAPRT levels that were barely detectable and were highly sensitive to FK866 (Supplementary Fig. S6A-S6C). NAPRT overexpression rendered MIA PaCa2 virtually completely resistant to FK866 (Supplementary Fig. S6AS6C). In control MIA PaCa2 cells, 2-HNA caused some sensitization to low FK866 concentrations $(<10 \mathrm{nmol} / \mathrm{L})$, but at higher FK866 concentrations ( $\geq 10 \mathrm{nmol} / \mathrm{L}$ ), it had either no or minor activity (Supplementary Fig. S6B and S6C). Conversely, in NAPRT-overexpressing cells, 2-HNA caused a striking sensitization to FK866 in the entire range of FK866 concentrations tested, fully reverting the resistance to this agent caused by NAPRT overexpression (Supplementary Fig. S6B and S6C).

Thus, NAPRT chemical inhibition is a promising therapeutic strategy for sensitizing NAPRT-overexpressing cancer cells to NAMPT inhibitors.

NAPRT silencing or NAPRT inhibition sensitizes ovarian cancer xenografts to FK866

To obtain the proof-of-concept that blocking NAPRT activity also sensitizes NAPRT-overexpressing cancer cells to a NAMPT inhibitor in vivo, we first generated subcutaneous xenografts of OVCAR-5, OVCAR-8, and Capan-1 cells transduced with scrshRNA or NAPRT-sh2 and evaluated their response to FK866 in terms of tumor volume and of tumor weight. In the case of OVCAR- 5 and of Capan-1 cells, only in subcutaneous xenografts with silenced NAPRT, but not in tumors expressing the control shRNA, FK866 reduced tumor growth (Fig. 6A-D). In OVCAR-8 xenografts, FK866 exerted some antitumor activity in both control and NAPRT-silenced tumors. However, the antitumor effect of FK866 was significantly more pronounced in the presence of NAPRT silencing (Fig. 6E-G). We also tested the activity of FK866 against subcutaneous xenografts of control and NAPRT-overexpressing MIA PaCa2. Again, the in vivo data resembled the in vitro results because the xenografts of control cells showed sensitivity to FK866, but NAPRT-overexpressing MIA PaCa2 were completely resistant (Supplementary Fig. S6D and S6E). Thereafter, we studied FK866's activity in a more clinically relevant model consisting in the intraperitoneal injection of OVCAR-5 (control cells and cells with silenced NAPRT) into nude mice (42). Using this model, we could not detect any effect of the NAMPT inhibitor on the 
A

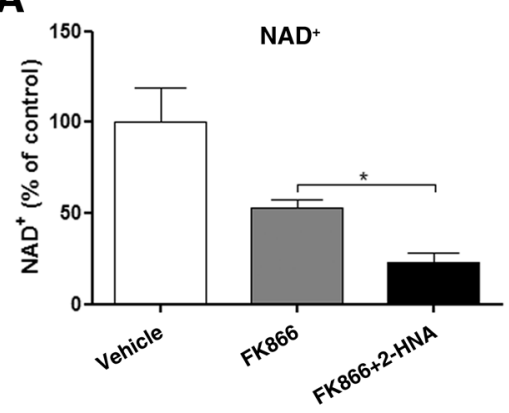

D

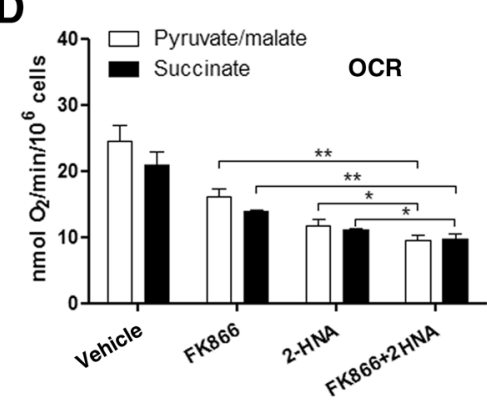

G

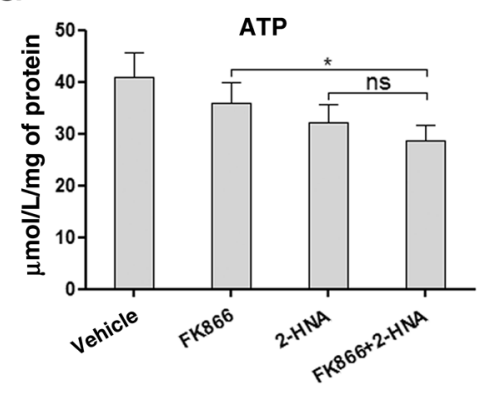

B

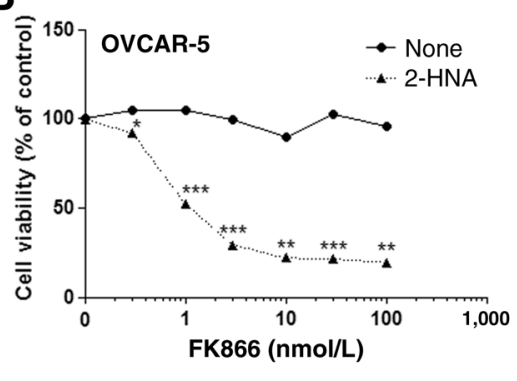

E

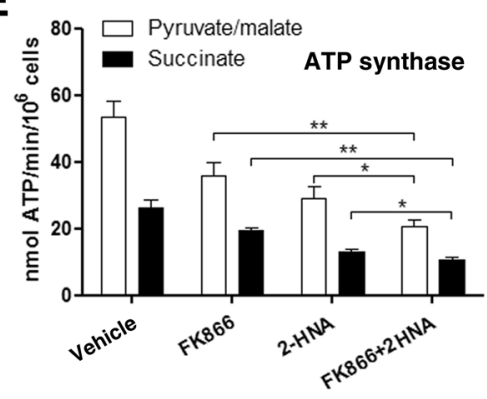

H

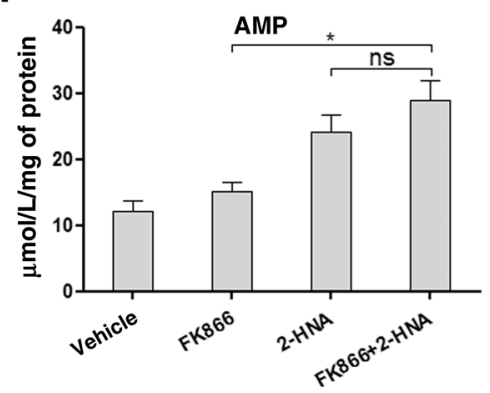

C

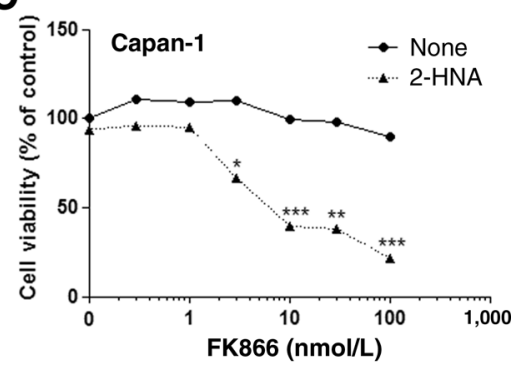

F

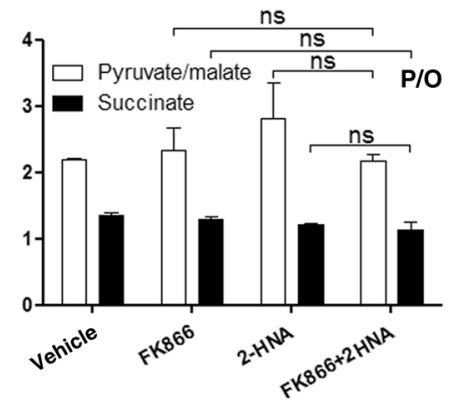

I

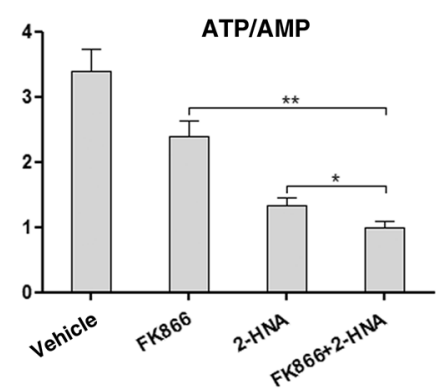

Figure 5.

The NAPRT inhibitor 2-HNA sensitizes cancer cells to FK866. A, $1 \times 10^{5}$ OVCAR-5 cells were seeded in 6-cm Petri dishes, allowed to adhere overnight, and

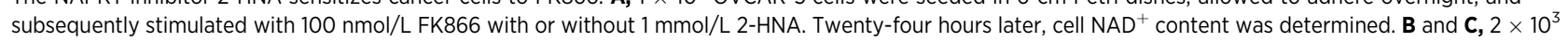
OVCAR-5 and Capan-1 cells/well were plated in 96-well plates, allowed to adhere overnight, and then incubated for 72 hours with or without FK866 at the indicated concentrations in the presence or absence of $1 \mathrm{mmol} / \mathrm{L} 2-\mathrm{HNA}$. Subsequently, cell viability was detected with SRB. D-I, $3 \times 10^{6}$ OVCAR- 5 cells/flask were seeded in $75 \mathrm{~cm}^{2}$ flasks, allowed to adhere overnight, and then stimulated for 24 hours with or without 100 nmol/L FK866 in the presence or absence of $1 \mathrm{mmol} / \mathrm{L}$ 2-HNA. Thereafter, cell lysates were generated and OCR, ATP synthase activity, ATP, AMP, as well as ATP/AMP and P/O ratios were determined. A, D-I, Data are presented as mean \pm SD of three separate experiments. B and C, Data are mean of three independent experiments. ns, nonsignificant; ${ }^{*}, P<0.05$; ${ }^{* *}, P<0.01$; ***, $P<0.001$. In A, D, E, G-I, the experimental values for FK866, 2-HNA, and FK866+2-HNA all had $P<0.05$ compared with those for vehicle.

survival of mice bearing control tumors (median survival of mice with tumors expressing the scr-shRNA treated with or without FK866 was 34.5 and 33 days, respectively; $P=0.45$; log rank test; Fig. $6 \mathrm{H}$ and I). On the contrary, we did observe a significant increase in mice survival when FK866 was used to treat mice bearing tumors with silenced NAPRT (median survival of mice with tumors expressing NAPRT-sh2 treated with or without FK866 was 45.5 and 37 days, respectively; $P=0.03$; log rank test; HR: 4.8). Mice with NAPRT-silenced tumors treated with FK866 also had a better survival than mice with control tumors (scr-shRNA) treated with or without the same agent $(P=0.012$ and $P=0.007$, respectively; HR: 6.9 and 8.9 , respectively). Interestingly, in mice bearing OVCAR-5 xenografts with silenced NAPRT, a trend toward an increase in survival compared with mice bearing control tumors (scr-shRNA) was also noticed, but it did not reach statistical significance $(P=0.14)$.

Given the poor solubility of 2-HNA, to be able to test the effect of NAPRT chemical inhibition on FK866's activity in vivo, we generated a sodium salt of 2-HNA itself (2-HNANa; Supplementary Fig. S7A), which indeed proved to be well-soluble in sterile saline and which we found to effectively sensitize OVCAR-5 to FK866 in culture (Supplementary Fig. S7B). We intraperitoneally injected mice with OVCAR-5 cells and 3 days after tumor inoculation assigned them to be treated for 3 weeks with either vehicle, FK866, or the combination of FK866 with 2-HNANa. Median survival of these treatment groups was 35.5, 33.5, and 42 days, respectively, with a statistically significant increase in survival versus the control group observed with the combination 
Piacente et al.
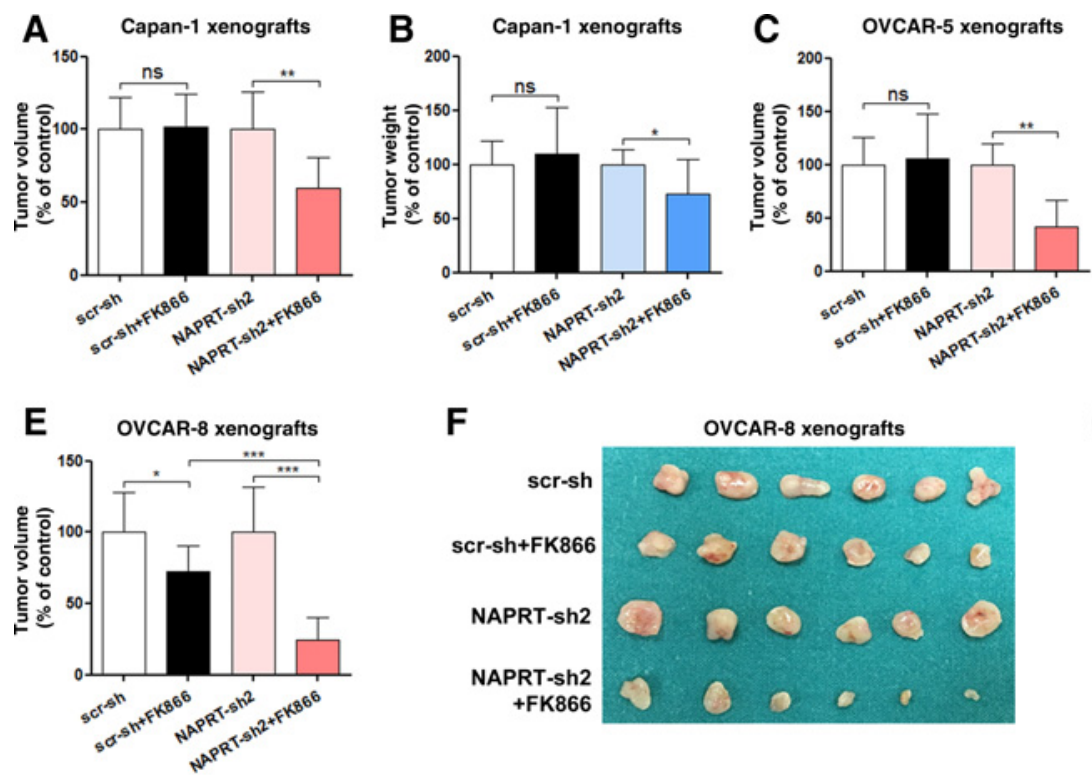

$\mathbf{F}$

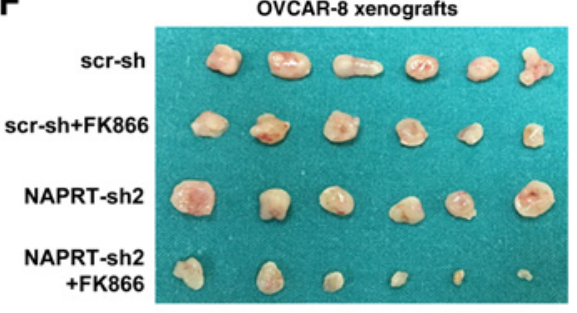

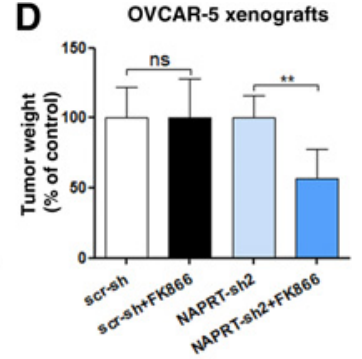

G

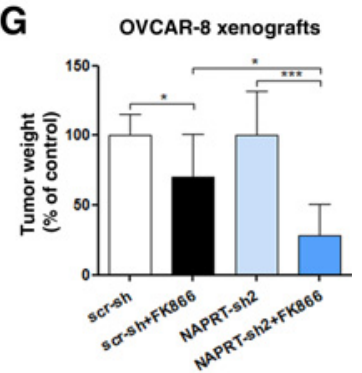

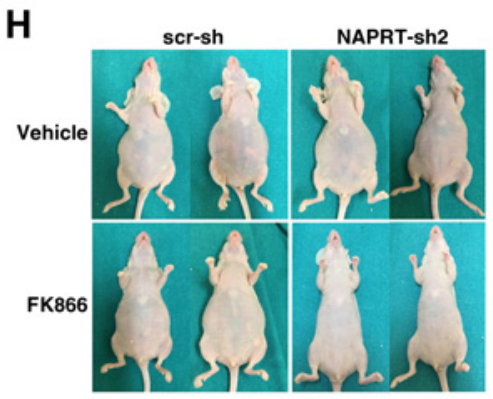

1

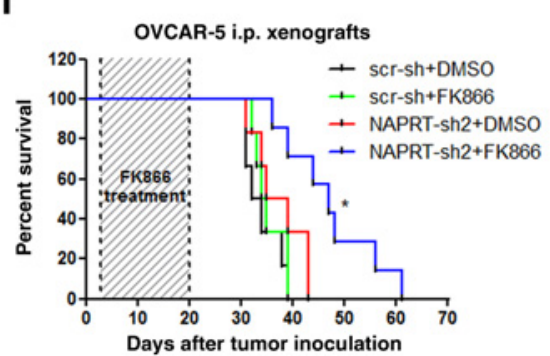

\section{$\mathbf{J}$}
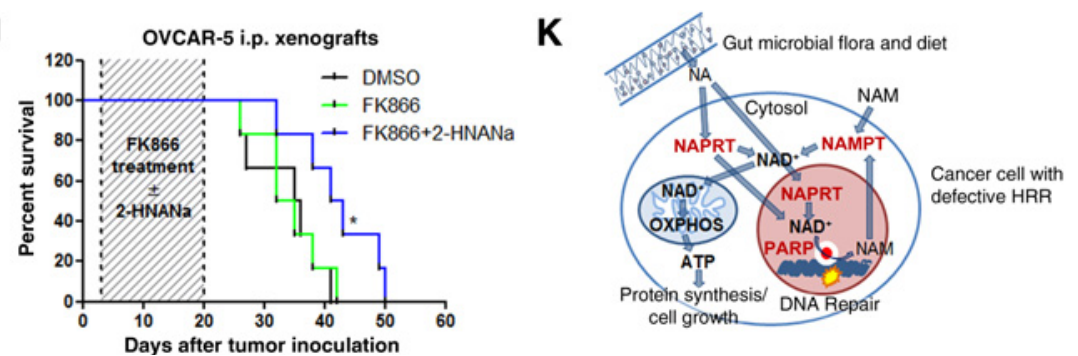

Figure 6.

NAPRT silencing and the NAPRT inhibitor 2-HNANa sensitize NAPRT-expressing cancer xenografts to FK866. A-G, $2 \times 10^{6}$ Capan-1 cells or $5 \times 10^{6}$ OVCAR-8 or OVCAR-5 cells engineered to express a control scr-shRNA or NAPRT-sh2 were injected subcutaneously into nude mice. When the tumors appeared as established palpable masses, mice ( $n=6$ per treatment arm) were randomly assigned to receive either vehicle or FK866 for 3 weeks. Tumor volume was monitored (A, C, and E), and at sacrifice, tumor masses were excised, photographed (F), and weighted (B, D, and G). In NAPRT-sh2-expressing Capan-1, OVCAR-5, and OVCAR-8 xenografts, FK866 reduced tumor volume by $40 \%, 59 \%$, and $75 \%$, respectively, and tumor weight by $27 \%$, $44 \%$, and $71 \%$, respectively. ns, nonsignificant; ${ }^{*}, P<0.05 ; * *, P<0.01 ; * * *, P<0.001$. H and I, Athymic mice were injected intraperitoneally with OVCAR-5 cells engineered with either a scr-shRNA or NAPRT-sh2. Three days after tumor inoculation, mice ( $n=8$ per treatment arm) were randomly assigned to be treated with either FK866 or vehicle DMSO for a total of 3 weeks. H, Mice were imaged 31 days after tumor inoculation (FK866 effectively delayed ascites development only in mice with NAPRT-silent tumors). I, Mice survival is shown. ${ }^{*}, P<0.05$ for NAPRT-sh2+FK866 versus NAPRT-sh2+DMSO (log-rank test). J, Athymic mice were injected i.p. with OVCAR-5 cells. Three days after tumor inoculation, mice ( $n=8$ per treatment arm) were randomly assigned to be treated with FK866, FK866+2-HNANa, or vehicle DMSO for a total of 3 weeks. Mice survival is shown. ${ }^{*}, P<0.05$ for FK866+2-HNANa versus FK866 alone (log-rank test). K, Putative model for NAPRT's role in cancer cells. NAPRT and NAMPT provide two parallel routes for NAD ${ }^{+}$production. While NAPRT primarily utilizes exogenous NA, whose primary source in vertebrates are the gut microbial flora and the diet, NAMPT utilizes both exogenous nicotinamide (NAM) and NAM produced inside the cells by NAD ${ }^{+}$degrading enzymes, such as PARPs, CD38, and sirtuins. NAPRT- and NAMPT-derived NAD ${ }^{+}$replenishes cytosolic, nuclear, and mitochondrial NAD ${ }^{+}$pools and supports the activity of DNA repair enzymes, such as PARP1/2. In NAPRT-overexpressing cancer cells, NAPRT-dependent NAD ${ }^{+}$biosynthesis appears to be especially important in promoting OXPHOS, in the maintenance of the cellular energy status, in the regulation of protein synthesis and of cell size, and in supporting the DNA repair process. 
FK866+2-HNANa $(P=0.033$; long rank test; HR: 4.9$)$, but not with FK866 alone $(P=0.77$; Fig. $6 \mathrm{~J})$.

\section{Discussion}

This is the first study to show (i) that the NAPRT gene is amplified and overexpressed in a subset of common types of cancer; (ii) that NAPRT plays a role in cancer cell metabolism, energy status, protein synthesis, cell size regulation, and DNA repair; and (iii) that chemical NAPRT inhibition overcomes resistance to NAMPT inhibitors previously tested in clinical trials.

Previous studies mainly focused on NAPRT as an enzyme, whose absence from cancer cells could make cancer cells exquisitely sensitive to NAMPT inhibitors and possibly also allow increasing the therapeutic window of these drugs by coadministration of NA (although the value of the latter strategy has recently been questioned; refs. 33, 43-48). Indeed, NAPRT expression appears to be down regulated (primarily by NAPRT gene promoter hypermethylation) in some types of cancer, such as softtissue malignancies, glioblastomas, and neuroblastomas $(33,47)$. Nevertheless, no study has ever highlighted the fact that NAPRT is actually amplified and resultantly overexpressed in subsets of other common and frequently lethal types of cancer (i.e., ovarian, pancreatic, prostate, breast, liver, and head and neck cancers), where it likely promotes cell metabolism and DNA repair and reduces susceptibility to NAMPT inhibitors and to DNA-damaging drugs.

Hara and colleagues showed that in human cells, NAPRT is essential for NA to increase cellular $\mathrm{NAD}^{+}$levels and that this enzyme can protect cells from the toxicity of $\mathrm{H}_{2} \mathrm{O}_{2}$ (34). Our study attributes new important functions to NAPRT, showing that in NAPRT-overexpressing cancer cells, NAPRT also contributes to the maintenance of the intracellular $\mathrm{NAD}^{+}$pool in baseline conditions. Interestingly, in Capan-1 cells, the cytosolic, but not the mitochondrial, $\mathrm{NAD}^{+}$pool was found to be resistant to NAPRT silencing, suggesting a specific reliance of mitochondria on NAPRT for the replenishment of their $\mathrm{NAD}^{+}$stores. We also demonstrate that cancer cells, in which NAPRT is silenced or inhibited, exhibit a marked reduction in their OXPHOS and in their ATP/AMP ratio, likely as a result of a reduction in their levels of mitochondrial $\mathrm{NADH}$, which is produced from $\mathrm{NAD}^{+}$and is required for the activity of complex I of the electron transport chain. The metabolic changes resulting from NAPRT deletion were found to translate into a reduction in protein synthesis and, ultimately, into a smaller cell size.

In epithelial ovarian cancer, we found a correlation between high-NAPRT expression and a BRCAness gene expression profile (27), and, consistent with these data, we were able to show a role for NAPRT in the DNA repair process of the BRCA-2deficient cell line, Capan-1. Overall, it seems plausible that, in ovarian cancer (and, possibly, also in other types of cancer) with defective HRR, NAPRT amplification and the resulting NAPRT overexpression could confer a selective advantage as they ensure that sufficient $\mathrm{NAD}^{+}$is produced to support PARP activity and also to protect cells from oxidative damage (34). In line with the hypothesis that defective $\mathrm{NAD}^{+}$synthesis is responsible for the increased DNA damage observed upon NAPRT silencing in Capan-1, we found that exogenously added $\mathrm{NAD}^{+}$reduced DNA damage in NAPRT-silenced cells treated with MMS. However, interestingly, this effect was much smaller compared with the effect of $\mathrm{NAD}^{+}$supplementation in control cells, where $\mathrm{NAD}^{+}$addition virtually abolished MMS-induced DNA damage. These data are not anticipated based on the current understanding of $\mathrm{NAD}^{+}$biosynthetic routes (38), because exogenous $\mathrm{NAD}^{+}$is thought to be degraded to yield nicotinamide riboside, nicotinamide mononucleotide, or nicotinamide (but not NA), and the latter precursors should be then reconverted into $\mathrm{NAD}^{+}$intracellularly independent of NAPRT (38). Thus, future studies should address a possible contribution of NAPRT to $\mathrm{NAD}^{+}$biosynthesis also starting from such precursors.

Several points support the notion that NAPRT amplification and its consequent overexpression represent a relevant mechanism of resistance to NAMPT inhibitors in humans. These include (i) the fact that NAPRT amplification is relatively common in human cancers, (ii) the resistance of human, NAPRT-overexpressing ovarian and pancreatic cancer cells to NAMPT inhibitors coupled to the fact that such resistance is effectively abolished by NAPRT-silencing, (iii) the presence of detectable NA levels in bodily fluids (49), and (iv) the fact that cancer xenografts were sensitized to the anticancer effects of FK866 if their NAPRT was either silenced or inhibited, whereas tumor xenografts that are normally sensitive to FK866 became resistant when overexpressing NAPRT (MIA PaCa2 model). The latter findings indicate that tumor NAPRT can indeed also have access to its substrate and act as a resistance mechanism to FK866 in vivo. The use of NAPRT inhibitors appears as a promising strategy to overcome NAPRT-mediated resistance to NAMPT inhibitors in patients. Several compounds with inhibitory activity on NAPRT were previously identified, such as 2 HNA, nucleotides, nucleosides, and intermediates of carbohydrates metabolism $(20,39-41)$. In our hands, 2-HNA proved effective. However, because this compound has a low potency as a NAPRT inhibitor, new NAPRT inhibitors will likely have to be identified.

In conclusion, our study shows that NAPRT is overexpressed in a subset of human cancer, where it contributes to the maintenance of the $\mathrm{NAD}^{+}$stores and to promote OXPHOS, ATP production, protein synthesis, and DNA repair (Fig. 6K). Targeting NAPRT could prove useful for sensitizing NAPRT-expressing cancer to NAMPT inhibitors and, possibly, to DNA-damaging agents. Our findings broaden our understanding of the function of the $\mathrm{NAD}^{+}$ biosynthetic apparatus in cancer cells and may facilitate the rational design of therapeutic strategies.

\section{Disclosure of Potential Conflicts of Interest}

No potential conflicts of interest were disclosed.

\section{Authors' Contributions}

Conception and design: F. Piacente, I. Caffa, A. Nahimana, M. Duchosal, A. Nencioni

Development of methodology: A. Nencioni

Acquisition of data (provided animals, acquired and managed patients, provided facilities, etc.): F. Piacente, I. Caffa, S. Ravera, M. Passalacqua, V.G. Vellone, P. Becherini, D. Reverberi, S. Bruzzone

Analysis and interpretation of data (e.g., statistical analysis, biostatistics, computational analysis): F. Piacente, I. Caffa, S. Ravera, V.G. Vellone, P. Becherini, F. Monacelli, P. Odetti, A. Cagnetta, M. Cea, S. Bruzzone, A. Nencion

Writing, review, and/or revision of the manuscript: F. Piacente, F. Monacelli, A. Nahimana, A. Nencioni

Study supervision: A. Ballestrero, P. Odetti, A. Nencioni

Other (Production of some of the data reported in the article): G. Sociali 


\section{Acknowledgments}

The authors wish to thank Dr. Nobumasu Hara (Shimane University Faculty of Medicine) for providing valuable reagents and Dr. Gabriella Cirmena (DIMI University of Genoa) for the excellent technical assistance.

\section{Grant Support}

This work was supported in part by the Associazione Italiana per la Ricerca sul Cancro (AIRC, \#6108, \#17736 to A. Nencioni), by the Seventh Framework Program projects PANACREAS (\#256986; to A. Nencioni) and ATHERO-BCELL (\#602114 to A. Nencioni), by the Fondazione Umberto Veronesi
(A. Nencioni and I. Caffa); by the Italian Ministry of Health (GR-2011 02347192 to A. Nencioni); by the $5 \times 10002014$ Funds to the IRCCS San Martino-IST (A. Nencioni and M. Cea), by the IBSA Foundation (F. Piacente); and by the University of Genoa.

The costs of publication of this article were defrayed in part by the payment of page charges. This article must therefore be hereby marked advertisement in accordance with 18 U.S.C. Section 1734 solely to indicate this fact.

Received November 17, 2016; revised April 6, 2017; accepted May 12, 2017 published OnlineFirst May 15, 2017.

\section{References}

1. Hanahan D, Weinberg RA. Hallmarks of cancer: the next generation. Cell 2011;144:646-74.

2. Garten A, Schuster S, Penke M, Gorski T, de Giorgis T, Kiess W. Physiological and pathophysiological roles of NAMPT and NAD metabolism. Nat Rev Endocrinol 2015;11:535-46.

3. Lerrer B, Gertler AA, Cohen HY. The complex role of SIRT6 in carcinogenesis. Carcinogenesis 2016;37:108-18.

4. Schiewer MJ, Knudsen KE. Transcriptional roles of PARP1 in cancer. Mol Cancer Res 2014;12:1069-80.

5. Vaisitti T, Audrito V, Serra S, Buonincontri R, Sociali G, Mannino E, et al. The enzymatic activities of CD38 enhance CLL growth and trafficking: implications for therapeutic targeting. Leukemia 2015; 29:356-68.

6. Cea M, Cagnetta A, Adamia S, Acharya C, Tai YT, Fulciniti M, et al. Evidence for a role of the histone deacetylase SIRT6 in DNA damage response of multiple myeloma cells. Blood 2016;127:1138-50.

7. Liu T, Liu PY, Marshall GM. The critical role of the class III histone deacetylase SIRT1 in cancer. Cancer Res 2009;69:1702-5.

8. Montecucco F, Cea M, Bauer I, Soncini D, Caffa I, Lasiglie D, et al. Nicotinamide phosphoribosyltransferase (NAMPT) inhibitors as therapeutics: rationales, controversies, clinical experience. Curr Drug Targets 2013;14:637-43.

9. Shackelford RE, Mayhall K, Maxwell NM, Kandil E, Coppola D. Nicotinamide phosphoribosyltransferase in malignancy: a review. Genes Cancer 2013;4:447-56.

10. Galli U, Travelli C, Massarotti A, Fakhfouri G, Rahimian R, Tron GC, et al Medicinal chemistry of nicotinamide phosphoribosyltransferase (NAMPT) inhibitors. J Med Chem 2013;56:6279-96.

11. Nahimana A, Attinger A, Aubry D, Greaney P, Ireson C, Thougaard AV, et al The NAD biosynthesis inhibitor APO866 has potent antitumor activity against hematologic malignancies. Blood 2009;113:3276-86.

12. Hasmann M, Schemainda I. FK866, a highly specific noncompetitive inhibitor of nicotinamide phosphoribosyltransferase, represents a novel mechanism for induction of tumor cell apoptosis. Cancer Res 2003; 63:7436-42.

13. Olesen UH, Christensen MK, Bjorkling F, Jaattela M, Jensen PB, Sehested M, et al. Anticancer agent CHS-828 inhibits cellular synthesis of NAD. Biochem Biophys Res Commun 2008;367:799-804.

14. von Heideman A, Berglund A, Larsson R, Nygren P. Safety and efficacy of NAD depleting cancer drugs: results of a phase I clinical trial of CHS 828 and overview of published data. Cancer Chemother Pharmacol 2010;65:1165-72

15. Grozio A, Sociali G, Sturla L, Caffa I, Soncini D, Salis A, et al. CD73 protein as a source of extracellular precursors for sustained NAD+ biosynthesis in FK866-treated tumor cells. J Biol Chem 2013;288: 25938-49.

16. Sociali G, Raffaghello L, Magnone M, Zamporlini F, Emionite L, Sturla L, et al. Antitumor effect of combined NAMPT and CD73 inhibition in an ovarian cancer model. Oncotarget 2016;7:2968-84.

17. Bogan $\mathrm{KL}$, Brenner $\mathrm{C}$. Nicotinic acid, nicotinamide, and nicotinamide riboside: a molecular evaluation of $\mathrm{NAD}+$ precursor vitamins in human nutrition. Annu Rev Nutr 2008;28:115-30.

18. Soncini D, Caffa I, Zoppoli G, Cea M, Cagnetta A, Passalacqua M, et al Nicotinamide phosphoribosyltransferase promotes epithelial-to-mesenchymal transition as a soluble factor independent of its enzymatic activity. J Biol Chem 2014;289:34189-204.

19. Caffa I, D'Agostino V, Damonte P, Soncini D, Cea M, Monacelli F, et al. Fasting potentiates the anticancer activity of tyrosine kinase inhibitors by strengthening MAPK signaling inhibition. Oncotarget 2015;6 11820-32.

20. Galassi L, Di Stefano M, Brunetti L, Orsomando G, Amici A, Ruggieri S, et al. Characterization of human nicotinate phosphoribosyltransferase: kinetic studies, structure prediction and functional analysis by site-directed mutagenesis. Biochimie 2012;94:300-9.

21. Lescuyer P, Strub JM, Luche S, Diemer H, Martinez P, Van Dorsselaer A, et al Progress in the definition of a reference human mitochondrial proteome Proteomics 2003;3:157-67.

22. Bruzzone S, Fruscione F, Morando S, Ferrando T, Poggi A, Garuti A, et al Catastrophic NAD + depletion in activated T lymphocytes through Nampt inhibition reduces demyelination and disability in EAE. PLoS One 2009;4 e7897.

23. Ledesma-Fernandez E, Thorpe PH. Fluorescent foci quantitation for high-throughput analysis. J Biol Methods 2015;2:e22. doi: 10.14440/ jbm.2015.62.

24. Damonte P, Hodgson JG, Chen JQ, Young LJ, Cardiff RD, Borowsky AD Mammary carcinoma behavior is programmed in the precancer stem cell. Breast Cancer Res 2008;10:R50.

25. Yeung TL, Leung CS, Wong KK, Samimi G, Thompson MS, Liu J, et al TGF-beta modulates ovarian cancer invasion by upregulating CAFderived versican in the tumor microenvironment. Cancer Res 2013;73 5016-28.

26. Subramanian A, Tamayo P, Mootha VK, Mukheriee S, Ebert BL, Gillette MA, et al. Gene set enrichment analysis: a knowledge-based approach for interpreting genome-wide expression profiles. Proc Nat Acad Sci USA 2005;102:15545-50.

27. Konstantinopoulos PA, Spentzos D, Karlan BY, Taniguchi T, Fountzilas E, Francoeur N, et al. Gene expression profile of BRCAness that correlates with responsiveness to chemotherapy and with outcome in patients with epithelial ovarian cancer. J Clin Oncol 2010;28:3555-61.

28. Cerami E, Gao I, Dogrusoz U, Gross BE, Sumer SO, Aksoy BA, et al. The cBio cancer genomics portal: an open platform for exploring multidimensional cancer genomics data. Cancer Discov 2012;2:401-4.

29. Gao J, Aksoy BA, Dogrusoz U, Dresdner G, Gross B, Sumer SO, et al. Integrative analysis of complex cancer genomics and clinical profiles using the cBioPortal. Sci Signal 2013;6:pl1.

30. Uhlen M, Fagerberg L, Hallstrom BM, Lindskog C, Oksvold P, Mardinoglu A, et al. Proteomics. Tissue-based map of the human proteome. Science 2015;347:1260419.

31. Uhlen M, Oksvold P, Fagerberg L, Lundberg E, Jonasson K, Forsberg M, et al. Towards a knowledge-based human protein atlas. Nat Biotech 2010;28:1248-50.

32. Moghaddas Gholami A, Hahne H, Wu Z, Auer FJ, Meng C, Wilhelm M, et al. Global proteome analysis of the NCI-60 cell line panel. Cell Rep 2013;4:609-20.

33. Watson M, Roulston A, Belec L, Billot X, Marcellus R, Bedard D, et al. The small molecule GMX1778 is a potent inhibitor of NAD+ biosynthesis: strategy for enhanced therapy in nicotinic acid phosphoribosyltransferase 1-deficient tumors. Mol Cell Biol 2009;29:5872-88.

34. Hara N, Yamada $K$, Shibata $T$, Osago $H$, Hashimoto $T$, Tsuchiya $M$. Elevation of cellular NAD levels by nicotinic acid and involvement of nicotinic acid phosphoribosyltransferase in human cells. J Biol Chem 2007;282:24574-82. 
35. Michels J, Vitale I, Saparbaev M, Castedo M, Kroemer G. Predictive biomarkers for cancer therapy with PARP inhibitors. Oncogene 2014;33: 3894-907.

36. Goggins M, Schutte M, Lu J, MoskalukCA, Weinstein CL, Petersen GM, et al Germline BRCA2 gene mutations in patients with apparently sporadic pancreatic carcinomas. Cancer Res 1996;56:5360-4.

37. Asher G, Reinke H, Altmeyer M, Gutierrez-Arcelus $M$, Hottiger $M O$, Schibler U. Poly(ADP-ribose) polymerase 1 participates in the phase entrainment of circadian clocks to feeding. Cell 2010;142:943-53.

38. Nikiforov A, Dolle C, Niere M, Ziegler M. Pathways and subcellular compartmentation of NAD biosynthesis in human cells: from entry of extracellular precursors to mitochondrial NAD generation. J Biol Chem 2011;286:21767-78.

39. Gaut ZN, Solomon HM. Uptake and metabolism of nicotinic acid by human blood platelets. Effects of structure analogs and metabolic inhibitors. Biochimica et Biophysica Acta 1970;201:316-22.

40. Gaut ZN, Solomon HM. Inhibition of 7-14 C-nicotinic acid metabolism in the human blood platelet by anti-inflammatory drugs. Res Commun Chem Pathol Pharmacol 1970;1:547-52.

41. Gaut ZN, Solomon HM. Inhibition of nicotinate phosphoribosyltransferase in human platelet lysate by nicotinic acid analogs. Biochem Pharmacol 1971;20:2903-6.

42. Molpus KL, Koelliker D, Atkins L, Kato DT, Buczek-Thomas J, Fuller AF Jr. et al. Characterization of a xenograft model of human ovarian carcinoma which produces intraperitoneal carcinomatosis and metastases in mice. Int J Cancer 1996;68:588-95.
43. Cerna D, Li H, Flaherty S, Takebe N, Coleman CN, Yoo SS Inhibition of nicotinamide phosphoribosyltransferase (NAMPT) activity by small molecule GMX1778 regulates reactive oxygen species (ROS)-mediated cytotoxicity in a p53- and nicotinic acid phosphoribosyltransferase1 (NAPRT1)-dependent manner. J Biol Chem 2012;287:22408-17.

44. O'Brien T, Oeh J, Xiao Y, Liang X, Vanderbilt A, Qin A, et al. Supplementation of nicotinic acid with NAMPT inhibitors results in loss of in vivo efficacy in NAPRT1-deficient tumor models. Neoplasia 2013;15: 1314-29.

45. Beauparlant P, Bedard D, Bernier C, Chan H, Gilbert K, Goulet D, et al. Preclinical development of the nicotinamide phosphoribosyl transferase inhibitor prodrug GMX1777. Anti-cancer Drugs 2009;20:346-54.

46. Shames DS, Elkins K, Walter K, Holcomb T, Du P, Mohl D, et al. Loss of NAPRT1 expression by tumor-specific promoter methylation provides a novel predictive biomarker for NAMPT inhibitors. Clin Cancer Res 2013;19:6912-23.

47. Tateishi K, Wakimoto H, Iafrate AJ, Tanaka S, Loebel F, Lelic N, et al. Extreme vulnerability of IDH1 mutant cancers to NAD+ depletion. Cancer Cell 2015;28:773-84.

48. Duarte-Pereira S, Pereira-Castro I, Silva SS, Correia MG, Neto C, da Costa LT, et al. Extensive regulation of nicotinate phosphoribosyltransferase (NAPRT) expression in human tissues and tumors. Oncotarget 2016;7 $1973-83$.

49. Kirkland JB.Niacin status, NAD distribution and ADP-ribose metabolism Curr Pharm Design 2009;15:3-11. 


\section{Cancer Research}

The Journal of Cancer Research (1916-1930) | The American Journal of Cancer (1931-1940)

\section{Nicotinic Acid Phosphoribosyltransferase Regulates Cancer Cell Metabolism, Susceptibility to NAMPT Inhibitors, and DNA Repair}

Francesco Piacente, Irene Caffa, Silvia Ravera, et al.

Cancer Res 2017;77:3857-3869. Published OnlineFirst May 15, 2017.

Updated version Access the most recent version of this article at: doi:10.1158/0008-5472.CAN-16-3079

Supplementary Access the most recent supplemental material at:

Material http://cancerres.aacrjournals.org/content/suppl/2017/05/13/0008-5472.CAN-16-3079.DC1

Cited articles This article cites 47 articles, 18 of which you can access for free at: http://cancerres.aacrjournals.org/content/77/14/3857.full\#ref-list-1

Citing articles This article has been cited by 1 HighWire-hosted articles. Access the articles at: http://cancerres.aacrjournals.org/content/77/14/3857.full\#related-urls

E-mail alerts Sign up to receive free email-alerts related to this article or journal.

Reprints and To order reprints of this article or to subscribe to the journal, contact the AACR Publications Department at Subscriptions pubs@aacr.org.

Permissions To request permission to re-use all or part of this article, use this link http://cancerres.aacrjournals.org/content/77/14/3857.

Click on "Request Permissions" which will take you to the Copyright Clearance Center's (CCC) Rightslink site. 